[Highlight]

\title{
基于金属有机框架和碳纳米管的可折叠锂-硫电池
}

\author{
庄 林
}

(武汉大学化学与分子科学学院, 武汉 430072)

\section{Metal-Organic Framework/Carbon Nanotube-Based Foldable Lithium-Sulfur Battery}

\author{
ZHUANG Lin \\ (College of Chemistry and Molecular Sciences, Wuhan University, Wuhan 430072, P. R. China)
}

随着能源危机和环境污染的不断加剧, 以及 电子电动设备的飞速发展, 对低成本高能量的储 能体系的研究和开发一直受到广泛关注。锂-硫电 池具有能量密度高, 价格低廉, 环境友好等优 点, 是极具潜力的新一代储能体系 ${ }^{1,2}$ 。然而, 锂硫电池中活性物质导电性差, 中间产物多硫化物 的溶解和穿梭效应, 以及充放电过程中较大的体 积变化等问题, 导致其活性物质利用率低, 循环 寿命较差, 制约着其进一步发展 ${ }^{3,4}$ 。因此, 如何提 高活性物质导电性, 抑制聚硫化锂的穿梭效应, 并获得较高能量密度的硫正极是提高锂-硫电池性 能的核心问题, 而合理有效的硫正极结构设计是 改善锂一硫电池性能的关键技术 ${ }^{5,6}$ 。

最近浙江大学彭新生教授和林展教授等合作 开发了一种基于金属有机框架材料(MOFs)和碳纳 米管的多级孔结构柔性复合薄膜, 用于高性能可 折叠柔性锂一硫电池, 相关结果发表在 Nature Communications 杂志上 ${ }^{7}$ 。该设计利用自限制固态 转换法获得了碳纳米管对 MOFs 进行穿插并编织成 自支撑的多级孔结构的柔性电极, 利用MOFs 材料 的高孔隙率和孔尺寸效应, 以及金属活性中心对 聚硫离子的吸附作用, 有效束缚活性物质于 MOFs 孔内, 抑制其溶解损失和穿梭效应; 同时由于碳
纳米管的穿插作用, 使得电极具有良好的导电 性, 以及优异的结构稳定性, 有利于硫高效和持 久的电化学反应。所得电池表现出优异的循环性 能, 较高的硫载量 $\left(11.33 \mathrm{mg} \cdot \mathrm{cm}^{-2}\right)$, 以及高达 $1195 \mathrm{mAh} \cdot \mathrm{cm}^{-3}$ 的能量密度, 并且可在不同折叠程 度上获得稳定的能量输出, 为获得高能量密度和 柔性的锂-硫, 乃至锂离子、钠离子和锂空气电池 等提供了新的思路。

\section{References}

(1) Manthiram, A.; Fu, Y.; Chung, S. H.; Zu, C.; Su, Y. S. Chem. Rev. 2014, 114, 11751. doi: 10.1021/cr500062v

(2) Bruce, P. G.; Freunberger, S. A.; Hardwick, L. J.; Tarascon, J. M. Nat. Mater. 2012, 11, 19. doi: 10.1038/nmat3191

(3) Wild, M.; O'Neill, L.; Zhang, T.; Purkayastha, R.; Minton, G.; Marinescu, M.; Offer, G. J. Energy Environ. Sci. 2015, 8, 3477. doi: $10.1039 / \mathrm{c} 5 \mathrm{ee} 01388 \mathrm{~g}$

(4) Hagen, M.; Hanselmann, D.; Ahlbrecht, K.; Maça, R.; Gerber, D.; Tübke, J. Adv. Energy Mater. 2015, 5, 1401986. doi: 10.1002/ aenm.201401986

(5) Seh, Z. W.; Sun, Y.; Zhang, Q.; Cui, Y. Chem. Soc. Rev. 2016, 45, 5605. doi: 10.1039/c5cs00410a

(6) Ogoke, O.; Wu, G.; Wang, X.; Casimir, A.; Ma, L.; Wu, T.; Lu, J. J. Mater. Chem. A 2017, 5, 448. doi: 10.1039/c6ta07864h

(7) Mao, Y.; Li, G.; Guo, Y.; Li, Z.; Liang, C.; Peng, X.; Lin, Z. Nat. Commun. 2017, 8, 14628. doi: 10.1038/ncomms14628 